\title{
Management of perinatal depression with non-drug interventions
}

\author{
Sara LJohansen, Thalia K Robakis, Katherine Ellie Williams, Natalie L Rasgon
}

Stanford University School of Medicine, Stanford, CA 94305 5119, USA

Correspondence to: KEWilliams elliew@stanford.edu

Cite this as: BMJ 2019;364:1322 doi: 10.1136/bmj.1322

Series explanation: State of the Art Reviews are commissioned on the basis of their relevance to academics and specialists in the US and internationally. For this reason they are written predominantly by US authors

\begin{abstract}
Perinatal depression is a common disorder that has been associated with serious risks to mother and child. Recently, screening for depression in pregnant and postpartum women has increased, as has the development of new psychotherapy and non-drug treatment modalities. Matching patients to treatments can be challenging, and although research into personalized treatment of major depression in the general population has increased, no published guidelines focus on personalized treatment approaches to perinatal depression. In particular, guidelines on non-drug treatments are lacking. This review summarizes the evidence on personalized non-drug treatment of perinatal depression, how to incorporate patients' preferences, novel treatments under investigation, and the potential role of biomarkers in matching patients to treatment. The review provides recommendations for future research in personalized care of perinatal depression.
\end{abstract}

\section{Introduction}

Perinatal depression is a common and disabling disorder, affecting approximately $13 \%$ of women in the general population. ${ }^{1}$ Even higher prevalence rates of $25-30 \%$ occur in groups at high risk, such as socioeconomically disadvantaged women. ${ }^{2}$ Consequently, the importance of early recognition and treatment of perinatal depression has been increasingly recognized. Multiple organizations, including the American College of Obstetricians and Gynecologists, ${ }^{3}$ American Academy of Pediatrics, ${ }^{4}$ and most recently the US Preventive Services Task Force, ${ }^{5}$ have all recommended screening for perinatal mood disorders during pregnancy and post partum. Many different treatment options now exist for women who are diagnosed as having perinatal depression, including psychopharmacologic interventions, such as antidepressants and psychotherapeutic treatments. ${ }^{67}$

Although many treatment options exist, remission rates in treatment studies vary widely and the rate of resistance to treatment in perinatal depression is unknown. In the only systematic review of response to treatment in studies of perinatal depression, the overall weighted mean rate of remission of adequately treated women, based on 17 studies examining remission rates for women with postpartum depression treated with at least six weeks of an adequate dose of antidepressant or evidence based psychotherapy, was $51.2 \%$ (95\% confidence interval $48.6 \%$ to $53.8 \%$ ). ${ }^{8}$

Recently, a personalized medicine approach to treatment of major depression in non-pregnant patients has been investigated and advocated, ${ }^{9}$ as factors such as patients' preferences, clinical characteristics, and biomarkers have all been studied as predictors of response to treatment. ${ }^{910}$ Perinatal depression in particular needs an individualized approach to treatment, as research has shown that the disorder presents with heterogeneity of symptoms and phenotypes, including depression associated with severe anxiety or anhedonia. ${ }^{11}$ Whether the different phenotypes of perinatal depression correspond to differing genetic profiles or biomarkers remains to be understood.

Although personalized treatment of non-perinatal depression has integrated recent research on biomarkers and genetics, especially in the choice of antidepressants, ${ }^{12}$ our understanding of the genetics and biomarkers of perinatal mood disorders is still in the early stages. Some of the most frequently implicated genes, including 5-HTT, ${ }^{13}$ OXTR $,{ }^{14} \mathrm{COMT},{ }^{15} \mathrm{MAOA},{ }^{16}$ and $\mathrm{HSD} 11 \mathrm{~B} 1,{ }^{17}$ have been assessed in a recent systematic review. ${ }^{18} \mathrm{~A}$ large scale effort to decode the genetics of postpartum depression is under way, ${ }^{19}$ but results are not yet published. An effort to use genome-wide association techniques to identify genetic pathways that could be differentially regulated in postpartum depression, using a discovery cohort of 62 and a replication cohort of 24, identified 116 transcripts differentially expressed in pregnancy between women who developed postpartum depression and those who did not. ${ }^{20}$ These transcripts were related to pathways involving estrogen signaling, selenium and folic acid metabolism, focal adhesion, adipogenesis, and apoptosis.

An alternative effort to use differential methylation to identify gene pathways involved in postpartum depression isolated two genes involved in estrogen receptor signaling whose methylation status may be predictive of the development of postpartum depression. ${ }^{21} \mathrm{~A}$ patent on the two biomarkers from this methylation study is pending, ${ }^{21}$ but none of the available information on the genetics of perinatal depression has yet been used for the prediction or treatment of perinatal depression in clinical settings. 


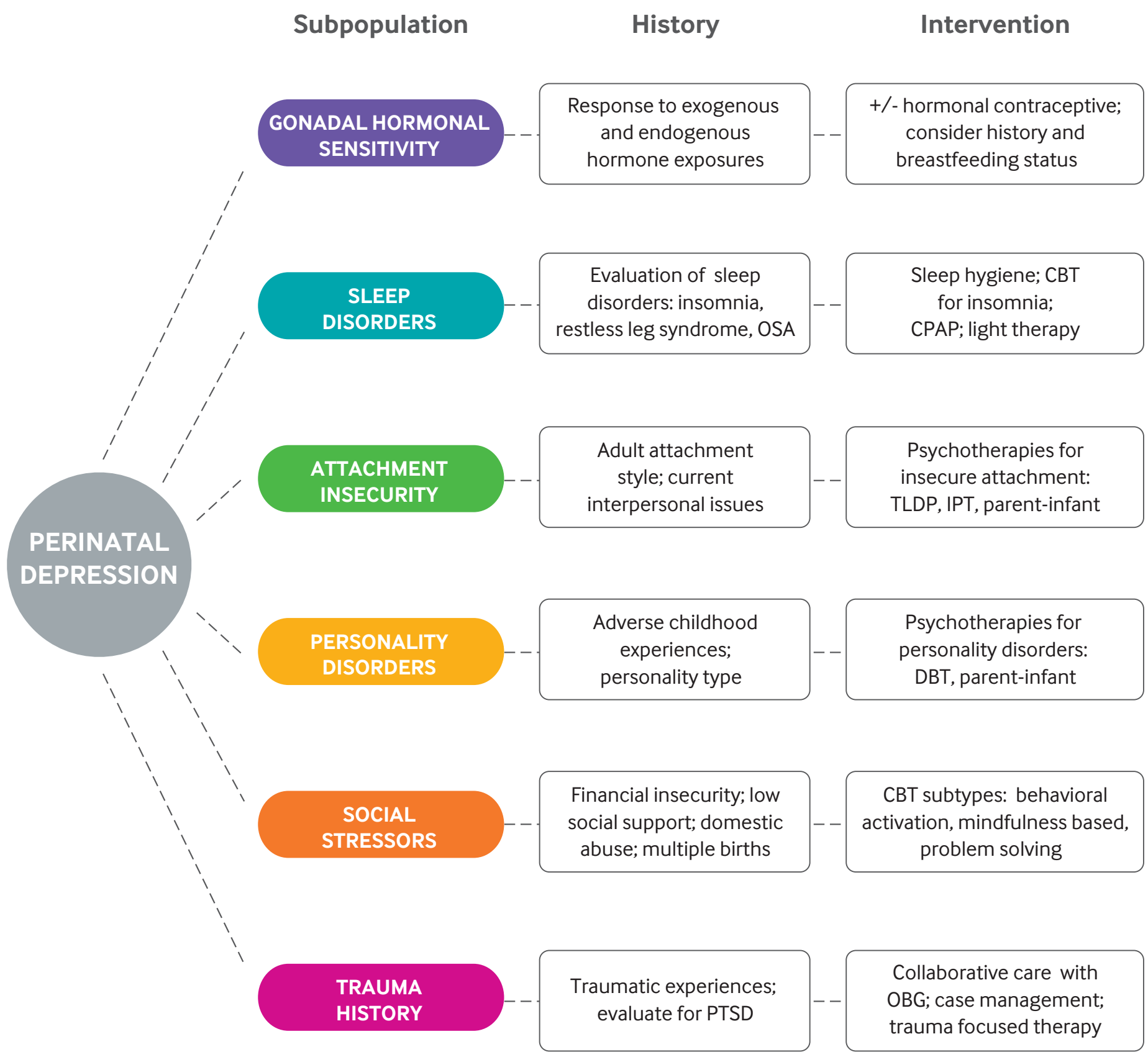

Personalized non-drug treatment recommendations for sub-populations of patients with perinatal depression based on clinical characteristics. $C B T=$ cognitive

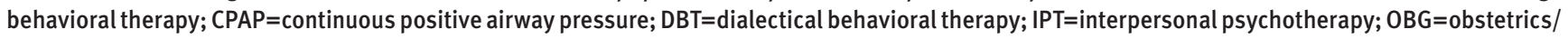
gynecology; OSA=obstructive sleep apnea; PTSD=post-traumatic stress disorder; TLDP=time limited dynamic psychotherapy

Thus, molecularly personalized perinatal depression treatment is still in its infancy, and this review will focus on the literature that will help to guide clinicians in their treatment choices, as specific patient populations may be more inclined to accept and/or benefit from certain treatments.

Although drug treatment is often central to the perinatal depression treatment plan, perinatal patients often prefer non-drug treatment. ${ }^{223}$ This review examines factors that influence the response to non-drug treatment in perinatal depression, focusing on how treatments may be tailored to subpopulations of patients with perinatal depression, including patients with gonadal hormone sensitivity, sleep disorders, attachment insecurity, personality disorders, social stressors, and trauma (figure).

\section{Sources and selection criteria}

We searched PubMed, Google Scholar, and Cochrane Reviews from January 2000 to January 2018 using the following MeSH search terms: treatment preferences, personalized medicine, psychotherapy, sleep disorders, attachment, personality disorders, trauma, cognitive behavior therapy, interpersonal psychotherapy, motherbaby units, transcranial magnetic stimulation (TMS), genetics, and postpartum depression or perinatal depression. We prioritized randomized controlled trials (RCTs), meta-analyses, longitudinal observational studies, and cohort studies. We included all full text, English language, peer reviewed publications of acceptable size and quality, with thresholds for size and quality depending on 
the range of data published for the specific topic. When large randomized trials were available, we did not discuss smaller case series or studies without control groups. We discussed pilot and observational work when large, well structured studies were not available. We identified additional studies by a hand search of references from articles identified by the original search. This review summarizes the most clinically relevant treatment information from the literature on this topic.

\section{Epidemiology of perinatal depression}

Perinatal depression is one of the most common morbidities of the perinatal period, affecting up to $14 \%$ of women in developed countries throughout pregnancy and up to a year after delivery, ${ }^{124}$ with approximately $19 \%$ of women having a depressive episode within the first three months post partum. ${ }^{1}$ The prevalence of perinatal depression is much higher in lower income and middle income countries, up to $20 \%$ on average. ${ }^{25}$ In the US, the prevalence of perinatal depression varies considerably across communities. Among 3952 Hispanic women interviewed in postpartum wards in Miami, New York, and San Francisco, $42.5 \%$ screened positive for depression. ${ }^{26} \mathrm{~A}$ sample of 192 inner city, financially impoverished African-American and European-American women found that $23.4 \%$ of women screened positive for postpartum depression, ${ }^{27}$ and $51 \%$ of a sample of 148 Hispanic and African-American pregnant women screened positive for depression ${ }^{28}$; of note, depression rates did not vary by ethnicity in either of these studies. ${ }^{27}{ }^{28}$ Social factors such as low social support, marital difficulties, negative life events, and intimate partner violence are known risk factors for depression in the perinatal period, ${ }^{29}$ which likely contribute to the variation in perinatal depression prevalence across different populations.

\section{Patients' preferences}

Evaluation and attention to patients' treatment preferences is now recognized as an integral component of personalized treatment of perinatal depression, as consideration of patients' preferences has been associated with increased adherence to treatment and indirect positive effects on treatment outcomes. ${ }^{30-32}$ Although many women receive antidepressants as first line treatment for depression, research has shown that many perinatal patients prefer non-drug treatments owing to fears that drugs will harm their baby. ${ }^{334}$

The largest study of perinatal treatment preferences to date included 509 pregnant women in their third trimester who were waiting for their obstetric appointments in a large urban medical center. ${ }^{22}$ In this convenience sample, $92 \%$ of women answered the questionnaire by stating that they preferred individual psychotherapy, and only $7 \%$ rated drug treatment as a first choice intervention. A limitation of the study is that it included predominantly white $(57 \%)$ or Latina (29\%) women who were married or living with a partner (89\%) and non-recent immigrant women of high socioeconomic status (81\%). Another study used a five point Likert-type scale to compare white and African-American pregnant women $(\mathrm{n}=108)$ who screened above 10 on the Edinburgh Postnatal Depression Scale (EPDS) regarding their confidence in perinatal treatment preferences. ${ }^{23}$ Although all women reported having the highest confidence in psychotherapy and/or support from family and friends, African-American women reported significantly less confidence in drug treatment than white women (mean score 2.42 v 3.04; $\mathrm{P}=0.01$ ). Furthermore, in a smaller survey based study $(n=60)$ of African-American women seeking routine prenatal care in a clinic for medically underserved residents, non-drug psychosocial treatments including mindfulness $(\mathrm{n}=30 ; \mathrm{P}<0.001)$ and interpersonal approaches $(\mathrm{n}=25$; $\mathrm{P}<0.001)$ were significantly preferred to drug treatment for prevention of postpartum depression. ${ }^{22}$

These studies show that as the evidence accumulates for non-drug treatments for perinatal depression, patients' preferences form a foundation for personalized treatment. Future research with large culturally and racially diverse populations is needed to improve understanding of patients' preferences in choice of perinatal depression treatment.

\section{Personalized treatment for specific clinical characteristics of perinatal depression}

Although this review will focus on personalizing the available non-drug treatments for perinatal depression, it is important to recognize that some non-psychiatric illnesses can produce or masquerade as perinatal depression and a variety of psychiatric disorders can produce similar presentations. All women with suspected postpartum depression should be screened for thyroid dysfunction..$^{35}$ Vitamin D deficiency has also been repeatedly associated with perinatal depression, ${ }^{36-38}$ as has, to a lesser extent, anemia. ${ }^{39}$ Given the frequency of both conditions in the general population, they are worth investigating in women with perinatal depression, despite the absence thus far of formal recommendations. Rare obstetric complications such as pituitary infarction, ${ }^{40}$ as well as commonplace phenomena such as dysphoric milk ejection reflex, substance misuse, and flares of pre-existing autoimmune disease can also present with symptoms of depression. ${ }^{41-43}$ Within psychiatry, care should be taken to disentangle unipolar depression from bipolar disorder, psychotic presentations, and postpartum flares of generalized anxiety and obsessive-compulsive disorder. Appropriate treatment selection begins, as always, with accurate diagnosis.

The ability to personalize non-drug treatment depends in large part on the individual clinical characteristics of the patient. Selection of appropriate treatment depends on comprehensive review of the relevant medical, obstetric, psychiatric, social, and trauma history. As detailed in figure 1, this review summarizes non-drug treatment options for subpopulations based on specific clinical characteristics, including women with gonadal hormonal sensitivity, sleep disorders, attachment insecurity, personality disorders, social stressors, and trauma. This review covers non-drug treatment, with particular focus on targeting psychotherapeutic approaches on the basis of clinical characteristics (table).

\section{Gonadal hormonal sensitivity}

Women with a history of gonadal hormonal sensitivity may be a subpopulation at risk for perinatal mood dis- 


\begin{tabular}{|c|c|c|c|c|c|c|c|c|c|c|c|}
\hline \multicolumn{12}{|c|}{ Patients' characteristics and psychotherapeutic approaches } \\
\hline \multirow[b]{2}{*}{ Characteristic } & \multicolumn{8}{|c|}{ Delivered in person } & \multicolumn{3}{|c|}{ Remotely delivered } \\
\hline & CBT & IPT & $\begin{array}{l}\text { Cognitive } \\
\text { processing therapy, } \\
\text { prolonged exposure }\end{array}$ & $\begin{array}{l}\text { Couples } \\
\text { therapy }\end{array}$ & $\begin{array}{l}\text { Sleep } \\
\text { hygiene, CBT } \\
\text { for insomnia }\end{array}$ & $\begin{array}{l}\text { Interpersonal } \\
\text { and social } \\
\text { rhythm therapy }\end{array}$ & $\begin{array}{l}\text { Dialectical } \\
\text { behavioral } \\
\text { therapy }\end{array}$ & $\begin{array}{l}\text { Parent-infant } \\
\text { psychotherapy }\end{array}$ & $\begin{array}{l}\text { Behavioral } \\
\text { activation }\end{array}$ & iCBT & $\begin{array}{l}\text { Telephone } \\
\text { IPT }\end{array}$ \\
\hline PTSD & $\star \star \star 44$ & $\star \star \star 45$ & $\star \star * 46$ & & & & & & & & \\
\hline Minority race or single motherhood & $\star \star \star \star 44$ & & & & & & & & & & \\
\hline Marital strain & & & & $\star 47$ & & & & & & & \\
\hline Attachment insecurity & & $\star \star \star \star 45$ & & & & & & $\star \star 48$ & & & \\
\hline Borderline personality disorder & & & & & & & *49 & $\star \star 48$ & & & \\
\hline Sleep disorder or insomnia & & & & & $\star \star \star 5051$ & & & & & & \\
\hline Bipolar disorder & & & & & & *十 & & & & & \\
\hline $\begin{array}{l}\text { Rural location, limited transportation, } \\
\text { or child care restrictions }\end{array}$ & & & & & & & & & $\star \star 52$ & $\star \star \star 53$ & $\star \star \star 5455$ \\
\hline \multicolumn{12}{|c|}{ 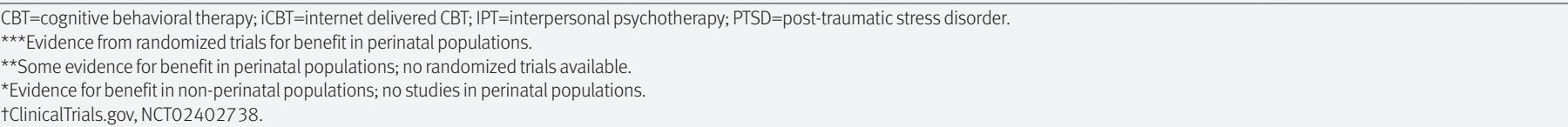 } \\
\hline
\end{tabular}

orders. Such sensitivities likely apply to only a subset, with most women not manifesting any particular mood disruption in relation to gonadal hormone fluxes. Thus, studies of unselected populations are unlikely to identify these women or their unique needs.

However, evidence supports the existence of women in whom gonadal hormone fluxes affect mood in important ways. In a study of the effects of rapid withdrawal of supraphysiologic concentrations of estrogen in women with and without a history of postpartum depression, five of eight women with postpartum depression developed significant depressive symptoms, compared with none of the women without this history. ${ }^{56}$ Studies attempting to correlate endogenous estrogen concentrations with mood symptoms in perinatal women have generally been negative, ${ }^{57}$ likely because the enormous effect of the psychosocial environment around pregnancy and delivery overshadows effects of steroids on mood in most women, and because mood variability is likely related to cellular target response to ovarian steroids and not to circulating steroid concentrations themselves. ${ }^{58}$

The only means of identifying women in this category is by taking a clinical history that specifically inquires about previous psychiatric responses to gonadal hormonal alterations, including menstrual cycles, hormonal contraceptives, and previous pregnancies. History of premenstrual dysphoric disorder (PMDD) may be an important clue, as some studies have found that women with PMDD are at elevated risk for postpartum depression, ${ }^{5960}$ and cellular responses to ovarian steroids are known to differ between women with PMDD and unaffected controls. ${ }^{58}$

Previous experience of mood disruption in response to hormonal contraceptives may also be an important element of the history. Although most women do not have onset of psychiatric symptoms with modern contraceptives, ${ }^{61}$ whether women who have a history of mood disorder are more sensitive to oral contraceptives remains controversial, as some reviews have suggested an increased risk of mood destabilization whereas others have not. ${ }^{6162}$ A nationwide prospective study of 1061997 women found that oral contraceptive users had higher rates of first diagnosis of depression (rate ratio $1.1,95 \%$ confidence interval 1.08 to 1.14 ) and antidepressant treatment (1.23, 1.22 to 1.25$)$ compared with non-users ${ }^{63}$; consequently, psychiatric response to oral contraceptives should be considered in postpartum women who are starting to use them. The 2016 United States Medical Eligibility Criteria for Contraceptive Use recommendation is that women use progesterone-only contraceptives rather than combined progesterone and estrogen preparations in the early postpartum period of up to 42 days and consider duration post partum and breastfeeding status when changing to preparations containing estrogen. ${ }^{64}$ However, one double blind, placebo controlled RCT in 180 women found that long acting progestogen contraceptive given within the first 48 hours of birth, compared with placebo injection can lead to increased risk of depressive symptoms as measured by the EPDS at six weeks post partum (EPDS $>11$; relative risk $1.75,1.12$ to 2.72$),{ }^{65}$ which may suggest that psychiatric response to progesterone contraceptives should be taken into account in the early postpartum period.

A "personalized" treatment approach to the group of reproductive hormone sensitive women with postpartum depression begins with a history of the woman's previous response to endogenous and exogenous hormone exposure, including oral contraceptives, gonadotropin releasing hormone agonists, estrogen replacement therapy, follicular stimulating drugs, or progesterone supplementation, and careful consideration of the postpartum hormonal milieu. Women who are breast feeding will have a unique reproductive hormone state of chronically low estrogen and progesterone without variation.

No studies have examined the effect of PMDD on psychiatric response to this hormonal state of breast feeding, but we could hypothesize that this absence of cyclical variation is favorable for these women, as women with PMDD report improved symptoms at other times of cycle cessation, such as after administration of gonadotropin releasing hormone agonist and with administration of extended cycle hormonal contraceptives. ${ }^{6667}$ Destabilization of mood may occur when menses resume; as this would be a time of increased risk for relapse, higher doses of antidepressant may be needed at that time. For other women, however, the low estrogen and progesterone state may not be favorable, and this potential phenotype 
should be considered in the differential diagnosis of treatment resistant perinatal depression. If the depression is severe, treatment resistant, and associated with suicidality, personalized treatment may include consideration of a shortened period of breast feeding and return to previous oral contraception if that was associated with mood stability.

Despite intense interest in past decades in the use of exogenous estrogen for the treatment of postpartum depression, evidence for the utility of this treatment is promising but remains preliminary, ${ }^{65}$ and it has not been widely adopted in clinical practice. Exogenous estrogen can interfere with lactation, ${ }^{68}$ which may represent a barrier to its adoption among breastfeeding women.

Neurosteroids, such as allopregnanolone, are allosteric modulators of the $y$-aminobutyric acid system. Allopregnanolone concentrations increase during pregnancy and fall rapidly post partum. Women with higher depression scores in late pregnancy have been found to have lower serum concentrations of allopregnanolone. ${ }^{69}$ Furthermore, in an exploratory study of 60 pregnant women with a previous diagnosis of mood disorder, higher concentrations of allopregnanolone during the second trimester were associated with a 63\% (95\% confidence interval $13 \%$ to $84 \%$ ) reduction in the risk of developing postpartum depression $(\mathrm{P}=0.02) .{ }^{70}$ Two double blind RCTs of a newly developed intravenous formulation of allopregnanolone, brexanolone (SAGE-547), have now been conducted. In the first placebo controlled trial in 138 women with postpartum depression who were admitted to the hospital for a single 60 hour intravenous injection of brexanolone at $90 \mu \mathrm{g} / \mathrm{kg} / \mathrm{h}$, brexanolone at $60 \mu \mathrm{g} / \mathrm{kg} / \mathrm{h}$, or matching placebo, the mean reduction in Hamilton Depression Rating Scale (HAM-D) scores was greater for both treatment groups, with 19.5 and 17.7 points in the brexanolone 60 and 90 groups, respectively, compared with 14.0 in the placebo group (difference $-5.5,-8.8$ to $-2.2(\mathrm{P}=0.013)$, brexanolone 60 group; $-3.7,-6.9$ to $0.5(\mathrm{P}=0.252)$, brexanolone 90 group). In the second study, 138 women were randomly assigned to receive brexanolone 90 or placebo. Mean reduction in HAM-D total score in this study was 14.6 points in the brexanolone group compared with 12.1 points for the placebo group (difference $-2.5,-4.5$ to $-0.5 ; \mathrm{P}=0.016$ ). ${ }^{71}$

Although widespread sampling of pregnant women for allopregnanolone concentrations is not indicated at this time, these recent findings from biomarker research and RCTs offer a novel potential personalized treatment for women with severe major depression resistant to conventional psychotherapeutic and psychopharmacologic modalities. This intervention is likely to be most appropriate for women with very severe postpartum depression as well as excellent social supports for infant care, as the drug is delivered by intravenous infusion and requires a hospital stay.

\section{Sleep disorders}

A bidirectional relation exists between perinatal mood disorders and sleep disorders. Problems with sleep are associated with onset of perinatal depression, and major depression is associated with sleep problems. ${ }^{72} 73$
Obstructive sleep apnea (OSA) is more common in pregnancy, ${ }^{73}$ and OSA is associated with an increased risk of gestational hypertension, intrauterine growth retardation, and gestational diabetes mellitus. ${ }^{73}$ OSA is associated with major depression in the general population; longitudinal studies show an increased risk of developing major depression in patients with OSA, and severity of OSA is related to the risk of developing major depression. ${ }^{74}$ Continuous positive airway pressure (CPAP) has been found to improve symptoms of depression in OSA patients with comorbid depression in some but not all studies of major depression in non-pregnant patients. ${ }^{7576}$

Despite the increased prevalence of OSA in perinatal patients, the prevalence of major depression in this specific subpopulation of sleep disordered perinatal patients remains unclear. To date, no studies of the efficacy of CPAP for comorbid depression in pregnant and postpartum patients have been conducted. Nevertheless, CPAP is indicated when OSA complicates pregnancy, ${ }^{73}$ and oral appliances for sleep apnea in pregnancy are under study as well (clinicaltrials.gov, NCT03646214 and NCT03138291). Consequently, a personalized treatment approach to treatment of perinatal depression in this population involves coordinated care with a sleep specialist to maximize adherence to and effectiveness of CPAP intervention.

Insomnia unrelated to OSA is also a risk factor for and a consequence of perinatal depression. ${ }^{73}$ Recent studies have also shown that poor sleep quality may be an independent risk factor for suicidal ideation among pregnant women. ${ }^{72}$ Women with documented sleep phase disorders, or with histories of seasonal affective disorder, constitute special populations who may find particular benefit from sleep based interventions. Postpartum weight retention has been associated with reduced sleep efficiency, ${ }^{77}$ suggesting that women with increased body mass or difficulty shedding pregnancy weight could also constitute a specific population of interest with respect to sleep based interventions.

Cognitive behavioral therapy (CBT) for insomnia has been shown to be effective in diverse psychiatric populations, including patients with major depression, posttraumatic stress disorder (PTSD), and anxiety disorders. ${ }^{78}$ Two small open pilots of CBT for insomnia in perinatal women have been published with encouraging preliminary results but small sample sizes and no control groups. ${ }^{5079}$ A randomized trial of a remotely delivered CBT for insomnia intervention for sleep disruption in pregnancy is ongoing. ${ }^{80}$

A less structured intervention for poor sleep in perinatal women is simply education on good sleep hygiene. A sleep plan that ensures the mother's ability to get longer blocks of uninterrupted night-time sleep, while still ensuring that the nutritional and care giving needs of the infant are met overnight, is a crucial component of treatment for postpartum depression in almost all cases. Most controlled trials of educational sleep interventions for postnatal dyads have focused on the quality of infants' sleep, and on balance these trials have not shown major benefits. ${ }^{81}$ Among the minority of trials that examined maternal depressive symptoms as an outcome, most stud- 
ies reported positive results. ${ }^{82-84}$ One notable pilot RCT of 30 postpartum women found that women who received a sleep counseling intervention averaged more nighttime sleep than controls (57 (95\% confidence interval 6 to 106) min; $\mathrm{P}=0.03) .{ }^{85}$ However, a larger RCT failed to replicate these results. ${ }^{86}$

Light therapy is also an important tool in promoting circadian eurhythmia and may be of particular importance in women who report worsening of mood in the winter months. Bright light therapy has shown superiority to dim light placebo for antenatal depression (remission attained by $68.6 \% v 36.4 \%$, respectively, as measured by the final HAM-D score after a five week treatment course; $\mathrm{P}<0.05) .{ }^{87}$ However, the only RCT for postpartum depression found no difference between women receiving light therapy and controls. ${ }^{88}$ This might be related to the significantly greater environmental circadian disruption induced by the need for overnight newborn care, which could potentially overwhelm any effect of light exposure.

An interesting recent advance in this area involves the use of home-wearable light devices, which reduce the burden of treatment associated with a stationary light box. Acceptability of a wearable light therapy device in postpartum women has been demonstrated, ${ }^{89}$ and a larger randomized trial is ongoing (clinicaltrials.gov, NCT02769858).

\section{Attachment insecurity}

People are prepared for successful parenthood by their own experiences of having been cared for as children. Childhood experiences have a major impact on a person's experience of parenthood and on adult attachment style (a facet of personality, shaped by early life experience, that structures adult interpersonal relationships). ${ }^{90-92}$ The quality of a woman's relationships with her own parents is a known risk factor for mood disruption in the perinatal period ${ }^{9394}$; in one study of 88 women who completed a structured diagnostic interview in the third trimester, women who recalled maternal "affectionless control" were seven times more likely to be diagnosed as having postpartum depression (odds ratio 6.8, 1.80 to 25.37; $\mathrm{P}<0.005){ }^{93}$

A personalized approach to perinatal depression should include an assessment of the patient's attachment style, including secure, anxious, and avoidant attachment styles, and if the patient seems to have insecure attachment, psychotherapies that are based in attachment theory may be particularly useful for this population. For instance, time limited dynamic psychotherapy (TLDP) is a short term psychotherapy based on the idea that people engage in maladaptive relationship patterns that lead to emotional distress, and these patterns are based on internal working models of relationships that are derived from childhood experiences. ${ }^{95}$ One study evaluated changes in attachment style over the course of manualized TLDP in 29 non-perinatal patients $(77 \%$ female; $96 \%$ white).${ }^{96}$ Patients evaluated before and after treatment with an interview based attachment rating system showed a significant increase in secure attachment themes identified by trained raters in videotaped narratives from before to after therapy (means for secure (2.09,
3.41) and fearful $(5.43,4.04)$ themes before and after treatment were significantly different $(\mathrm{P}<0.05)){ }^{96}$

Interpersonal psychotherapy is another treatment that could be beneficial in perinatal patients with interpersonal relationship problems due to attachment insecurity. Interpersonal psychotherapy is a dynamically informed psychotherapy that focuses on three key interpersonal themes-role transition, role disputes, and grief-and has been shown to be an effective treatment for perinatal depression, either as monotherapy or in conjunction with antidepressant. ${ }^{97}$ All depressed perinatal patients should be evaluated for underlying interpersonal problems that may contribute to the onset or persistence of depressive symptoms. Identification of interpersonal problems is a critical element of a personalized approach to treatment of perinatal depression, as the few studies that have examined risk factors for persistent depressive symptoms post partum have reported that ongoing relationship problems is a risk factor. ${ }^{98}$

\section{Personality disorders}

Personality disorders, which are also related to adverse experiences in childhood, ${ }^{33}$ have been less well studied in this population; nevertheless, available evidence indicates that pre-existing personality disorders may also represent risk factors for both anxiety and depression in pregnancy. ${ }^{99}$ In a sample of 34 women with depressive symptoms assessed with the EPDS at six weeks and one year post partum, those with postpartum depression persisting at one year (EPDS score $>12$ ) were significantly more likely to have an axis II disorder (odds ratio 21.1, 1.49 to $2.47 ; \mathrm{P}=0.02) .{ }^{100}$ Mothers with strong borderline and narcissistic traits or disorders often have difficulty with effective parenting, and studies suggest that their dysregulation of affect and difficulty with empathy and mentalizing may all have negative effects on child development. ${ }^{101}$ In a recent study, borderline personality disorder was found to be an independent risk factor for negative effects of perinatal depression on child development. ${ }^{102}$ Women with borderline personality disorder are also at increased risk for an array of adverse obstetric outcomes, ${ }^{103}$ including gestational diabetes (odds ratio 1.45 , 1.13 to 1.85$)$, premature rupture of membranes (1.40, 1.07 to 1.83$)$, chorioamnionitis $(1.65,1.14$ to 2.39$)$, venous thromboembolism $(2.11,1.12$ to 3.96$)$, cesarean delivery $(1.44,1.26$ to 1.64$)$, and preterm birth (1.54, 1.29 to 1.83$)$, emphasizing the importance of identifying and treating borderline personality disorder in pregnant women.

To date, no studies have identified the role of personality disorders in response to psychopharmacologic or psychotherapeutic interventions for perinatal depression. Given that personality disorders are known to be associated with resistance to treatment in non-depressed patients, ${ }^{104105}$ this is an important area of future research. A personalized approach to the patient with comorbid personality disorder and perinatal depression would include evidence based treatments for the personality disorder. For instance, in perinatal patients with borderline personality disorder, a dialectical behavioral therapy intervention should be considered, as this has been 
shown to be effective in reducing symptoms in patients with borderline personality disorder. Attachment therapies have been recommended, which include individual dynamically oriented psychotherapy focused on maternal childhood attachments and the association of maternal experiences with attachment figures to the current role as a mother. ${ }^{101}$ RCTs are needed to compare outcomes of mood and parenting in perinatal patients with personality disorder.

Parent-infant psychotherapy is another attachment oriented psychotherapy that represents an important personalized approach to treatment in patients with comorbid borderline personality disorder and perinatal depression. Parent-infant therapy addresses the difficulty that borderline and narcissistic patients have with mentalization of their infants. A promising method of parent-infant therapy is the watch, wait, and wonder method, ${ }^{106}$ which teaches mothers to observe and understand their infants' communication efforts and internal states. A case study of six mothers with borderline personality disorder describes successful implementation of this method, ${ }^{107}$ but no RCTs have looked at whether adjunctive attachment oriented psychotherapies enhance treatment for perinatal depression with comorbid personality disorders.

\section{Social stressors}

Several social stressors of particular relevance in the perinatal period have been shown to affect a woman's risk of developing depression or anxiety. These include younger age, lower level of education, lack of partner, poor social support, marital conflict, and domestic abuse. ${ }^{108-111}$ Other stressors unique to the perinatal period include unplanned pregnancy, a history of previous pregnancy losses, previous difficult pregnancy, or the delivery of a medically compromised infant. ${ }^{112113}$

CBT is a structured form of psychotherapy that focuses on identification and modification of negative or dysfunctional thought patterns and may be a particularly helpful intervention for women with significant social stressors. Its efficacy for both prevention and treatment of perinatal depression has been established in more than 40 RCTs, which have been assessed in multiple meta-analytic reviews. ${ }^{74114115}$ Subgenres of CBT that have been tested in perinatal populations include behavioral activation, ${ }^{116}$ mindfulness based cognitive therapy, ${ }^{117}$ and problem solving therapy. ${ }^{118}$ Common modifications for the perinatal population include the addition of peer support, ${ }^{119}$ partner involvement, ${ }^{120}$ and parenting education, ${ }^{121}$ all additions that may be helpful personalized psychotherapy interventions for women with social stressors.

The most recent meta-analysis of trials of CBT for perinatal women assessed which characteristics of the interventions might be associated with greatest benefit. ${ }^{44}$ It suggested that individual therapy might be associated with greater reductions in depressive symptoms than group therapy, and interventions targeted to later stages of pregnancy or the postpartum period were often more effective than those started earlier in pregnancy. Certain characteristics of patients such as single motherhood and minority race were associated with greater benefit from the intervention.

\section{Trauma}

PTSD is especially common in the perinatal period in high risk groups. In a systematic review and meta-analysis, the prevalence of PTSD in pregnancy was $34.4 \%$ for women with a history of childhood trauma and maltreatment. ${ }^{122}$ Recent studies have shown a high comorbidity between PTSD and perinatal depression in women with a history of trauma, ${ }^{123124}$ similar to the high comorbidity findings in the general population. ${ }^{125}$ Most studies of psychopharmacology and psychotherapy for perinatal treatment have not included patients with comorbid PTSD, and research is needed on providing effective treatment for perinatal depression with comorbid PTSD.

One promising personalized approach to perinatal depression with comorbid PTSD is the MOMCare collaborative care intervention, which includes medication management in collaboration with the patient's obstetrician/gynecologist; telephone and in-person delivery of brief interpersonal psychotherapy; case management and problem solving for basic needs such food, housing, and job training; and active outreach after missed sessions. ${ }^{126}$ This collaborative care model used an active stepped care approach, and patients who had less than $50 \%$ improvement in depressive symptoms by six to eight weeks received augmentation of interpersonal psychotherapy with drugs or increased dosage of drugs. A randomized study compared MOMCare treatment for 18 months of extended follow-up with maternity support services, which included referral to obstetrics and gynecology for psychopharmacologic intervention or outside services. ${ }^{126}$ For women with comorbid PTSD and perinatal depression, the MOMCare program $(\mathrm{n}=48)$, versus control $(n=58)$, was associated with significant improvement in severity of depression (Wald $\mathrm{c}^{2}=8.51 ; \mathrm{P}<0.05$ ), depression response (Wald $\mathrm{c}^{2}=4.13 ; \mathrm{P}<0.4$ ), and remission (Wald $\left.\mathrm{c}^{2}=8.07 ; \mathrm{P}<0.02\right){ }^{126}$ Women without comorbid PTSD experienced similar declines in depression severity, regardless of treatment group (Wald $\mathrm{c}^{2}=0.01 ; \mathrm{P}=0.90$ ).

An RCT of the brief interpersonal psychotherapy model $(n=21)$ versus enhanced treatment as usual $(n=21)$ in a low income population of pregnant women, reported that brief interpersonal psychotherapy was superior to enhanced treatment as usual in improving social support satisfaction (mean difference in Social Support Questionnaire Revised from baseline to 37-39 weeks' gestation -0.73 (95\% confidence interval -1.36 to -0.08 ) versus $0.63(-0.04$ to 1.26$)) .{ }^{127}$ However, no difference was seen between groups in reduction of depressive symptoms, as depression scores decreased significantly in both groups $(58 \%(n=11 / 19)$ and $63 \%(n=12 / 19)$ of participants in the brief interpersonal therapy and treatment as usual cohorts, respectively, reported reliable and clinically significant improvement in depressive symptoms from baseline to 37-39 weeks' gestation). Notably, in the enhanced treatment as usual group, 29\% received psychotherapy, $19 \%$ antidepressant drugs, and $1.5 \%$ drugs and counseling, and $43.5 \%$ received no mental health services, leading investigators to question whether this group was a useful comparator. ${ }^{127}$ Another explanation for the failure to replicate the earlier findings was the difficulty in providing interpersonal psychotherapy through various modali- 
ties, including consistent telephone based interpersonal psychotherapy or obstetric clinic based interpersonal psychotherapy, which are personalized treatments that have been found to be very useful in underserved populations with limited transportation and childcare support. ${ }^{128}$

Trauma focused psychotherapy by an experienced therapist is recommended for treatment of PTSD in the general population, ${ }^{129130}$ as well as in perinatal women. ${ }^{46}$ However, despite the prevalence of perinatal PTSD, no RCTs of trauma focused therapy in perinatal depression comorbid with PTSD have been conducted. Nevertheless, trauma focused therapy by an experienced therapist may be an important enhancement to treatment of depression in women with perinatal PTSD. Future studies should focus on the effectiveness of cognitive processing therapy and prolonged exposure, in addition to serotonin reuptake inhibitors, which have an evidence base for treating PTSD in the general population. ${ }^{131} 132$

\section{Personalized treatment approaches}

\section{Psychotherapy}

Neuroimaging studies in the general population have shown significant decreases in activation of the anterior cingulate/paracingulate gyrus and insula with psychotherapy that correspond to improved emotion regulation. ${ }^{133}$ The efficacy of interpersonal and cognitive behavioral psychotherapy for the treatment of perinatal depression has been extensively reviewed, ${ }^{134135}$ and several meta-analyses have reported that the effectiveness of psychotherapy in the perinatal population is similar to that in the general population. ${ }^{7136137}$

When recommending psychotherapy for perinatal depression, recent research on response to psychotherapy has shown the importance of considering patients' characteristics, such as attachment style, personality factors, social stressors and supports, and trauma history (table 1). Choice of psychotherapy in perinatal patients should evaluate and weigh the salience of specific symptoms and interpersonal factors. Not all psychotherapies will be effective for every patient. For instance, patients with comorbid anxiety disorders in general population studies were found to have decreased treatment response with interpersonal psychotherapy ${ }^{138}$; consequently, these patients may best be treated with CBT tailored to treatment of anxiety as well as depression.

PTSD has been found to be an important subpopulation in perinatal depression, and perinatal depression with comorbid PTSD may benefit from personalized treatments such as interpersonal psychotherapy. ${ }^{126}$ Additionally, women with bipolar disorder constitute a group at uniquely elevated risk for postpartum mood disorders, ${ }^{139}$ and whereas the mainstay of treatment for bipolar disorder consists of drugs, interpersonal and social rhythm therapy may be helpful adjuncts. ${ }^{140}$ No published studies have evaluated the utility of this therapy in pregnant women, but a randomized trial is in progress (clinicaltrials.gov, NCT02402738). Future studies of perinatal depression should identify subpopulations and comorbid disorders and evaluate whether interventions used for these disorders in non-pregnant patients enhance the effectiveness of treatment in the perinatal population.
Group support interventions may be particularly helpful for perinatal depression. A recent meta-analysis of seven RCTs involving 869 participants on group support for postpartum depression determined that this type of intervention was more effective than treatment as usual, with a pooled standardized mean difference of -0.59 ( -0.98 to $-0.21 ; \mathrm{P}=0.002)$. Among seven RCTs with 301 participants, peer support and group CBT were comparable in effect size, with a pooled standardized mean difference of $0.10(-0.20$ to $0.39 ; \mathrm{P}=0.53){ }^{141}$ These interventions may help to overcome challenges that are common to postpartum women, including infants' sleep and feeding routines, marital and family relationships, and general household disruption.

\section{Novel remote interventions}

Traditional psychotherapeutic treatments are time intensive, labor intensive, and expensive, requiring many hours of one-on-one attention from a trained specialist. Cost and time are common barriers to engaging in therapy, and even more so for women who are primary care givers for infants or young children. In recognition of this, interest in developing technology assisted methods for delivering the benefits of psychotherapy to perinatal women while mitigating problems of cost, time, and travel is growing.

Involvement of trained therapists in remote format therapy span a range from those that are entirely automated to remote delivery of traditional one-on-one therapy. In between are formats that combine automated provision of information or techniques with a variable amount of therapist and/or peer support. Automated formats are most adaptable to highly structured and manualized therapies. Remote provision of traditional individual psychotherapy can be adapted to most therapeutic modalities but does not provide appreciable improvements in cost or temporal flexibility over face-toface encounters. However, remote interventions can save the patient substantial time in travel to the therapist's office and provide the benefit of not having to leave young infants at home or take them out in public.

Remote interventions may be an important personalized approach to mothers of multiples who are especially burdened by logistical constraints. Women with multiples have been found to be at higher risk for postpartum depression than women of singletons; however, to date, no studies have focused on the efficacy of traditional psychotherapies in this population. In the first study to investigate unique mental health preferences of parents of multiples, 241 parents completed an online survey of treatment preferences and barriers; 53\% indicated that they would be interested in some form of technological treatment. ${ }^{142}$

\section{Internet based behavioral activation}

In one of the earliest published internet based interventions, an RCT randomly assigned 910 women with postpartum depression (EPDS $>12$ ) to an internet delivered behavioral activation program for postnatal depression versus treatment as usual. ${ }^{116}$ The intervention involved the delivery of 11 weekly sessions that included an educa- 
tional multimedia presentation and a homework exercise and offered access to an online chat room for peer support. Although only $38 \%$ (343/910) of participants completed the 15 week outcome assessment, fewer women in the intervention group continued to exceed the depression score cut-off (66/181) compared with treatment as usual $(91 / 162)$. This study was limited by high attrition rates but suggested that these types of interventions could offer benefit, although further research is needed.

\section{Computer and internet based CBT}

A recent meta-analysis of therapist supported, internet based CBT for postpartum women identified eight RCTs in this population..$^{53}$ Interventions ranged from six to 12 sessions and combined classical CBT approaches such as cognitive restructuring and behavioral activation with elements of psychoeducation, parenting preparation, and parenting focus that were specific to the postpartum period. All studies showed significant improvements over controls receiving treatment as usual in symptoms of stress ( $d=0.84$; five studies), anxiety ( $d=0.36$; six studies), and depression ( $\mathrm{d}=0.63$; eight studies). No study included a comparison with classical face-to-face CBT, but effect sizes for such interventions have been found to be in a similar range. ${ }^{44}$ The authors noted that attrition was greater in interventions that combined self help with limited therapist support, compared with those with exclusive therapist support.

A previous meta-analysis analyzed 11 trials of computer based CBT for perinatal women more broadly. ${ }^{143} \mathrm{In}$ this analysis, effect sizes were smaller and results more mixed, particularly for stress and anxiety symptoms, although overall most studies showed some benefit, and results for depressive symptoms were robust. Interventions intended to address existing mental health concerns were more effective than preventive approaches.

Recruitment for these studies relied almost entirely on media advertisements and self referrals, so the results may not apply equally to clinical populations. Nevertheless, they are encouraging in their potential to reach women who may not come to clinical attention.

\section{Telephone based interpersonal psychotherapy}

Interpersonal psychotherapy is less structured than CBT and thus does not lend itself to computer based approaches. However, remote delivery of individual psychotherapy has the potential to be more accessible to women who are tasked with the constant and temporally inflexible demands of a newborn. A single prospective cohort study of telephone based interpersonal psychotherapy for postpartum depression has been published. ${ }^{54}$ Therapists were certified nurse midwives who received 16 hours of therapy training but otherwise had no previous training or experience as psychotherapists. For participants who received the intervention of eight 50 minute sessions $(\mathrm{n}=41)$, the HAM-D score at eight and 12 weeks was significantly lower compared with controls $(\mathrm{P}=0.047$ at week 8; $\mathrm{P}=0.029$ at week 12). Women in the intervention group found the intervention highly acceptable and more accessible than traditional face-toface psychotherapy; however, five out of eight therapists dropped out of the trial, suggesting that attrition may be high among non-specialists providing psychotherapy. Among the 20 control women referred to usual care, only 10 actually obtained any mental healthcare. This is quite typical and underlines the point that remotely delivered therapies often represent a feasible alternative, not to appropriately delivered in-person mental healthcare, but to no care at all. An ongoing RCT of telephone based interpersonal psychotherapy provided by trained nurses to 240 postpartum women has also presented positive initial results in abstract form,,$^{55}$ although publication of final results is still pending.

\section{Emerging treatments}

Because pregnant women constitute a vulnerable population and are thus not usually appropriate participants for trials of new drugs, most of the treatments available in this field have been piloted for safety and efficacy in the general population first. The notable exception to this is brexanolone, discussed in the section above on gonadal hormonal sensitivity, development of which arose from basic research implicating allopregnanolone in postpartum depression. Other than this intervention, in the future it will be important to look at two specific populations of perinatal women that are currently underserved: the large pool of women with mild to moderate illness who go undiagnosed and untreated, and the much smaller but more seriously impaired pool of women who have severe illness that does not respond to first line treatments.

\section{Technology assisted psychotherapy}

Treatments that hold promise for a broader reach among women who would otherwise have gone undiagnosed and untreated include the variety of technology assisted psychotherapies discussed above. These range from remote delivery of classical individual psychotherapy to app based interventions with the capacity to reach unlimited numbers of women. Remotely delivered psychotherapies have the potential to provide flexibly available mental healthcare that can be accessed around the needs of a newborn and to reach women who otherwise would not come to mental health treatment owing to problems of stigma, accessibility, or cost. Although most studies have been conducted in non-clinical populations, the available evidence suggests that most remotely delivered therapies are superior to treatment as usual. Future studies in perinatal depression should investigate whether these novel psychotherapies increase engagement in therapy and represent a personalized approach to psychotherapy that is effective. Much work remains to be done to document the efficacy and applicability of each of these interventions, but as a whole they hold great promise for addressing the enormous problem of underidentification and undertreatment of perinatal mood disruption.

\section{Specialized perinatal units}

Treatments that hold promise for women in the severe or treatment resistant category include the establishment of specialized perinatal units and mother-baby units. Women with severe perinatal mental illness may need to be admitted to hospital to achieve stability in a safe 
environment. Specialized perinatal units have been an important treatment option for women in other nations for many years, ${ }^{144145}$ and they are now being offered in the US. ${ }^{146} 147$ Established across countries in Europe, psychiatric mother-baby units allow for infants to remain on the psychiatric unit with their mother 24 hours a day. ${ }^{144145}$ Such units have not been possible in the US owing to the healthcare system that does not support neonatal or pediatric care in the psychiatry unit; however, specialized perinatal units have been established that allow for infants to be on the unit for extended hours during the day. ${ }^{147}$ These inpatient perinatal units offer specialized care with particular attention to maternal-infant attachment.

In their evaluation of the first inpatient perinatal unit in the US, Meltzer-Brody et al reported a significant decrease in EPDS scores between the time of admission and time of discharge in their sample of 91 patients, two thirds of whom completed both sets of questionnaires. ${ }^{147}$ The mean admission EPDS was 20.23 (SD 5.57), and the mean discharge EPDS was 10 (5.50), for a significant mean difference of -10.29 (95\% confidence interval -11.93 to -8.65; $\mathrm{P}<0.001 ; \mathrm{n}=65 ; \mathrm{t}$ ratio=-12.53). Anxiety scores on the Generalized Anxiety Disorder questionnaire also decreased significantly; the mean score was 14.48 (5.52) at admission and 7.54 (5.15) at discharge, for a significant difference of -7.69 ( -9.29 to $-6.08 ; \mathrm{P}<0.001 ; \mathrm{n}=63$; t ratio $=-9.58)$. The authors did not report the number of women taking drug treatment or the types of drugs, but the description of the program emphasized the use of multiple non-drug treatments, including individual and group psychotherapy that included mindfulness, acceptance, and commitment therapy components, an attachment group led by a parent-infant specialist, and a weekly therapy group for partners and family. It is notable that $87 \%$ of the sample endorsed thoughts of self harm on the EPDS at admission. As many of the published studies of drug and psychotherapy treatment exclude women with suicidal ideation, studies of the effectiveness of treatment in severely ill depressed women with suicidal ideation are needed. Personalized treatment options for severely ill women can also include outpatient perinatal units, which are growing in number in the US and offer similar multimodal treatment interventions. ${ }^{146}$

\section{Transcranial magnetic stimulation}

An additional treatment that is established in the general population with major depression and that also has potential for use in perinatal women is TMS, which is approved by the US Food and Drug Administration for patients who have failed to respond to at least one antidepressant. TMS is particularly relevant to the pregnant population because it has the potential to avoid pharmacologic effects on the fetus, and it is significantly less invasive than the other available neurostimulatory approaches such as electroconvulsive therapy and vagal nerve stimulation.

Two open label pilot studies of TMS for pregnant women have been reported. ${ }^{148149}$ Among 10 women with major depressive disorder in the second or third trimester who received 20 sessions of $1 \mathrm{~Hz}$ TMS at 100\% motor threshold to the right dorsolateral prefrontal cortex, seven women responded to treatment, defined as greater than $50 \%$ reduction in HAM-D score. ${ }^{148}$ In a second pilot study of 30 women with depression in pregnancy, patients received 18 sessions of $25 \mathrm{~Hz}$ TMS at $100 \%$ motor threshold to the left dorsolateral prefrontal cortex, and approximately $40 \%$ of participants had greater than $50 \%$ reduction in HAM-D score; in both of these studies, the procedure was generally well tolerated, most women achieved some response, and approximately one third achieved remission. No adverse pregnancy or fetal outcomes were reported in either study.

Several case reports and small case series also report good tolerability of TMS in pregnant women with depression. ${ }^{150-152}$ Garcia and colleagues report remission in eight of nine women treated with TMS for postpartum depression. ${ }^{153}$ One estimate of likely fetal exposure to electrical fields in maternal TMS concluded that exposure would likely be well below the threshold of potential for harm. ${ }^{154}$ Some evidence also exists for using electroconvulsive therapy in patients who do not respond to TMS. ${ }^{155}$

Overall, TMS is a viable option for pregnant or postpartum patients with depression. Its clinical efficacy is comparable to that of drugs, ${ }^{156}$ while avoiding their systemic effects. Although it would be optimal to have more data on TMS for pregnant patients, and specifically on long term developmental outcomes, the available information at least suggests that the procedure is generally well tolerated. From a safety perspective, TMS is a reasonable choice, although it should be understood that TMS is not likely to represent a panacea. One major drawback is the frequency of treatment needed (typically daily for a period of many weeks), which would likely prove a significant barrier to women with childcare responsibilities.

\section{Clinical trials}

Thirty six trials are listed on clinicaltrials.gov under the heading of perinatal depression and treatment. Several recently completed or ongoing (result reporting dates pending) studies include innovative models of care delivery focused on breaking down barriers to care, such as virtual treatments with video conference visits with patients staying within their own home (clinicaltrials. gov, NCT03291600; estimated completion date March 2019). Multiple studies are investigating novel alterations of standard, evidence based psychotherapy treatments, such as enhancement of interpersonal psychotherapy with interpersonal psychotherapy with the mother-infant dyad (NCT03499756; completed January 2017) or couples (NCT03499756, estimated completion date December 2020). Sleep interventions are another area of focus in emerging treatment trials, and studies include bright light therapy (NCT02053649; estimated completion date February 2018; NCT02664467; estimated completion date November 2019) and positive airway pressure for depressed pregnant women with disordered breathing (NCT02507297; estimated completion date June 2020).

\section{Guidelines}

In terms of diagnosing perinatal depression, the US Preventive Services Task Force, American College of Obstetricians and Gynecologists, and American Academy of 


\section{GLOSSARY OF ABBREVIATIONS}

CBT-cognitive behavioral therapy
CPAP-continuous positive airway pressure
EPDS-Edinburgh Postnatal Depression Scale
HAM-D-Hamilton Depression Rating Scale
OSA-obstructive sleep apnea
PMDD-premenstrual dysphoric disorder
PTSD-post-traumatic stress disorder
RCT-randomized controlled trial
TLDP-time limited dynamic psychotherapy
TMS-transcranial magnetic stimulation

\section{SUGGESTIONS FOR FUTURE RESEARCH}

- Use of evidence based treatments for personality disorders in the general population, such as dialectic behavioral therapy for borderline personality, in women with perinatal psychiatric disorders

- Efficacy of transcranial magnetic stimulation in perinatal depression, including follow-up studies in infants

- Comparison of the efficacy of remote therapies and in-office psychotherapies in perinatal populations

Pediatrics recommend screening with a validated tool such as the EPDS during pregnancy and postpartum. ${ }^{34}$ Although screening is recommended, universal screening is not a required component of standard care. Furthermore, in practice, a single screening is not sufficient to identify women with perinatal depression, as depression can occur at any time during the pregnancy and postpartum.

Most guidelines on the treatment of perinatal depression focus on drug treatment. To date, few published guidelines cover non-drug treatment approaches to perinatal depression. A recent international analysis of clinical practice guidelines for treatment of perinatal depression found that among 16 international guidelines, most guidelines, including the American Psychiatric Association (US), Centre of Perinatal Excellence (Australia), and National Institute for Health and Care Excellence (UK), agree that psychotherapy, and in particular CBT, should be used as initial treatment in mild to moderate depression during pregnancy. ${ }^{157}$ Most guidelines indicate that an approach focused on antidepressant drug treatment should be used in severe depression in pregnancy. ${ }^{157}$ Only the American College of Obstetricians and Gynecologists determined that antidepressants should be used as initial treatment instead of psychotherapy during pregnancy, independent of severity of depression. In the postpartum period, most guidelines agree that psychotherapy should be the initial treatment for mild to moderate depression, with consideration of antidepressants as initial treatment for severe depression, and most encourage continued breast feeding, independent of the type of antidepressant drug. ${ }^{157}$ Of note, these guidelines favor a personalized approach to treatment, based on detailed history that allows for identification of clinical characteristics to guide individualized treatment.

\section{Limitations and future directions}

A major limitation to our understanding of how best to personalize treatment for perinatal mood disorders is our

\section{HOW PATIENTS WERE INVOLVED IN THE CREATION OF THIS}

\section{REVIEW}

We interviewed patients who presented for consultation and treatment of perinatal depression during the writing of this review. We did not do formal interviews with patients, and patients did not view or edit the content of the manuscript. The goal of these interviews was to evaluate patients' preferences for information on non-drug interventions in the treatment of perinatal depression. Patients expressed desire to understand which non-drug interventions, including psychotherapy and non-psychotropic drug interventions, were most likely to help perinatal depression; the differences between types of psychotherapy; and which non-drug therapies best matched their symptom profile.

incomplete understanding of the basic biologic mechanisms for mood disruption associated with gonadal steroids. Although the recent identification of cellular mechanisms such as expression of the estrogen effector complex ESC/E(Z) represents an important forward stride, ${ }^{58}$ our understanding of this area remains limited. Future work in this area will be important to establish some means beyond clinical history of identifying women at risk for mood disruption in relation to alterations in reproductive hormone concentrations.

Another area in which our understanding remains limited is in the conception of the genetic and epigenetic factors that shape mood response in the perinatal period. Although genetic personalization of drug therapies is an established reality in many areas of medicine, the field of perinatal psychiatry is still at the stage of attempting to identify potentially relevant genes and their associated molecular pathways. Much work remains to be done to form a molecularly based understanding of perinatal mood disruption that would be sufficient to inform selection of patients or treatment alternatives.

The absence of specific investigation of personality disorders and history of early life adversity with respect to psychotherapeutic interventions in postpartum women is also an important limitation of our current state of knowledge. A history of early life adversity clearly contributes to risk for perinatal mood disruption, and personality disorders are independent risk factors for negative effects of perinatal depression on child development, but current recommendations for treatment approaches in women with these risk factors have largely been drawn from work in non-perinatal populations. Studies specifically designed to investigate the unique needs of perinatal women would be a welcome and important guide for clinicians working in this field.

\section{Conclusions}

Perinatal depression is a heterogeneous disorder that may result from a wide variety of medical, social, and psychiatric impairments. Although psychopharmacologic interventions are an important aspect of treatment, nondrug interventions also play a key role in this population and importantly are preferred by pregnant and postpartum women owing to concerns about transplacental and lactational transfer of drugs.

Appropriate non-drug interventions include sleep management, group support, individual therapy, remote 
and computer assisted interventions, and more intensive interventions such as transcranial magnetic stimulation for pregnant women and specialized mother-baby units for postpartum women. Decisions about how to determine which of these would be most beneficial for a given woman will depend on the particular nexus of psychiatric, psychosocial, and medical factors that contribute to her illness, as well as on her own expressed preferences. More research is needed on personalized treatment approaches to help to match patients to treatments. Future studies should focus on clinical factors involved in response to traditional and novel psychotherapies, potential biomarkers for treatment choices, and treatment options for treatment resistant patients.

Contributors: SLJ, TKR, KEW, and NLR contributed equally to this work in terms of research, writing, generation of figures and tables, and revisions to the manuscript.

Competing interests: We have read and understood BMJ policy on declaration of interests and declare the following interests: none.

Provenance and peer review: Commissioned; externally peer reviewed.

1 Gavin NI, Gaynes BN, Lohr KN, Meltzer-Brody S, Gartlehner G, Swinson T. Perinatal depression: a systematic review of prevalence and incidence. Obstet Gynecol 2005;106:1071-83. 10.1097/01. AOG.0000183597.31630 db pmid:16260528.

2 Hobfoll SE, Ritter C, Lavin J, Hulsizer MR, Cameron RP. Depression prevalence and incidence among inner-city pregnant and postpartum women./ Consult Clin Psychol 1995;63:445-53. 10.1037/0022 006X.63.3.445 pmid.7608357.

3 Committee on Obstetric Practice. The American College of Obstetricians and Gynecologists Committee Opinion no. 630. Screening for perinatal depression. Obstet Gynecol 2015;125:1268-71.10.1097/01 AOG.0000465192.34779.dc pmid:25932866.

4 Earls MF. Committee on Psychosocial Aspects of Child and Family Health American Academy of Pediatrics. Incorporating recognition and management of perinatal and postpartum depression into pediatric practice. Pediatrics 2010;126:1032-9.10.1542/peds.20102348 pmid:20974776

5 O'Connor E, Rossom RC, Henninger M, Groom HC, Burda BU. Primary Care Screening for and Treatment of Depression in Pregnant and Postpartum Women: Evidence Report and Systematic Review for the US Preventive Services Task Force. JAMA 2016;315:388-406. 10.1001/ jama.2015.18948 pmid:26813212.

6 Chisolm MS, Payne JL. Management of psychotropic drugs during pregnancy. BMJ 2016;532:h5918. 10.1136/bmj. h5918 pmid:26791406.

7 Sockol LE, Epperson CN, Barber JP. A meta-analysis of treatments for perinatal depression. Clin Psychol Rev 2011;31:839-49.10.1016/j. cpr.2011.03.009 pmid:21545782.

8 Cox EQ, Sowa NA, Meltzer-Brody SE, Gaynes BN. The Perinatal Depression Treatment Cascade: Baby Steps Toward Improving Outcomes. / Clin Psychiatry 2016;77:1189-200. 10.4088/ ICP.15r10174 pmid:27780317.

9 Prendes-Alvarez S, Nemeroff CB. Personalized medicine: Prediction of disease vulnerability in mood disorders. Neurosci Lett 2018;669:10-3. 10.1016/j.neulet.2016.09.049 pmid:27746310.

10 Strawbridge R, Arnone D, Danese A, Papadopoulos A, Herane Vives A, Cleare AJ. Inflammation and clinical response to treatment in depression: A meta-analysis. Eur Neuropsychopharmacol 2015;25:1532 43.10.1016/j.euroneuro.2015.06.007 pmid:26169573.

11 Putnam KT, Wilcox M, Robertson-Blackmore E, et al. Postpartum Depression: Action Towards Causes and Treatment (PACT) Consortium. Clinical phenotypes of perinatal depression and time of symptom onset: analysis of data from an international consortium. Lancet Psychiatry 2017;4:477-85. 10.1016/S2215-0366(17)30136-0 pmid:28476427.

12 Amare AT, Schubert KO, Baune BT. Pharmacogenomics in the treatment of mood disorders: Strategies and Opportunities for personalized psychiatry. EPMA/ 2017;8:211-27. 10.1007/s13167-017-01128 pmid:29021832.

13 Zhang X, Wang L, Huang F, et al. Gene-environment interaction in postpartum depression: a Chinese clinical study. J Affect Disord 2014;165:208-12.10.1016/j.jad.2014.04.049 pmid:24882202.

14 Jonas W, Mileva-Seitz V, Girard AW, et al. MAVAN Research Team. Genetic variation in oxytocin rs 2740210 and early adversity associated with postpartum depression and breastfeeding duration. Genes Brain Behav 2013;12:681-94.pmid:23941164.

15 Comasco E, Sylvén SM, Papadopoulos FC, SundströmPoromaa I, Oreland L, Skalkidou A. Postpartum depression symptoms: a case-control study on monoaminergic functional polymorphisms and environmental stressors. Psychiatr Genet 2011;21:19-28. 10.1097/ YPG.0b013e328341a3c1 pmid:21099450.
16 Doornbos B, Dijck-Brouwer DA, Kema IP, et al. The development of peripartum depressive symptoms is associated with gene polymorphisms of MAOA, 5-HTT and COMT. Prog Neuropsychopharmacol Biol Psychiatry 2009:33:1250-4. 10.1016/i.pnpbp.2009.07.013 pmid:19625011.

17 Iliadis SI, Comasco E, Hellgren C, Kollia N, Sundström Poromaa I, Skalkidou A. Associations between a polymorphism in the hydroxysteroid (11-beta) dehydrogenase 1 gene, neuroticism and postpartum depression. J Affect Disord 2017;207:141-7. 10.1016/j. jad.2016.09.030 pmid:27721188.

18 Figueiredo FP, Parada AP, de Araujo LF, Silva WA Ir., Del-Ben CM. The Influence of genetic factors on peripartum depression: A systematic review. J Affect Disord 2015;172:265-73. 10.1016/j. jad.2014.10.016 pmid:25451426.

19 PPD ACT. Postpartum Depression: Action Towards Causes and Treatment. 2017. http://www.pactforthecure.com/.

20 Mehta D, Newport DJ, Frishman G, et al. Early predictive biomarkers for postpartum depression point to a role for estrogen receptor signaling. Psychol Med 2014;44:2309-22. 10.1017/ S0033291713003231 pmid:24495551.

21 Guintivano J, Arad M, Gould TD, Payne IL, Kaminsky ZA. Antenatal prediction of postpartum depression with blood DNA methylation biomarkers. Mol Psychiatry 2014;19:560-7.10.1038/ mp.2013.62 pmid:23689534.

22 Goodman JH. Women's attitudes, preferences, and perceived barriers to treatment for perinatal depression. Birth 2009;36:60-9. 10.1111/j.1523-536X.2008.00296.x pmid:19278385.

23 O'Mahen HA, Flynn HA. Preferences and perceived barriers to treatmen for depression during the perinatal period. J Womens Health (LarChmt) 2008;17:1301-9.10.1089/jwh.2007.0631 pmid:18816202.

24 Wisner KL, Sit DK, McShea MC, et al. Onset timing, thoughts of selfharm, and diagnoses in postpartum women with screen-positive depression findings. JAMA Psychiatry 2013;70:490-8. 10.1001/ jamapsychiatry.2013.87 pmid:23487258.

25 Fisher J, Cabral de Mello M, Patel V, et al. Prevalence and determinants of common perinatal mental disorders in women in low- and lowermiddle-income countries: a systematic review. Bull World Health Organ 2012:90:139G-49G. 10.2471/BLT.11.091850 pmid:22423165.

26 Kuo WH, Wilson TE, Holman S, Fuentes-Afflick E, O'Sullivan MJ, Minkoff H. Depressive symptoms in the immediate postpartum period among Hispanic women in three U.S. cities. J Immigr Health 2004;6:145-53. 10.1023/B:JOlH.0000045252.10412.fa pmid:16228697.

27 Hobfoll SE, Ritter C, Lavin J, Hulsizer MR, Cameron RP. Depression prevalence and incidence among inner-city pregnant and postpartum women. J Consult Clin Psychol 1995;63:445-53. 10.1037/0022 006X.63.3.445 pmid:7608357

28 Zayas LH, Cunningham M, McKee MD, Jankowski KR. Depression and negative life events among pregnant African-American and Hispanic women. Womens Health Issues 2002:12:16-22.10.1016/S10493867(01)00138-4 pmid:11786288.

29 Howard LM, Molyneaux E, Dennis CL, Rochat T, Stein A, Milgrom J. Non-psychotic mental disorders in the perinatal period. Lancet 2014;384:1775-88.10.1016/S0140-6736(14)612769 pmid:25455248.

30 Scholle SH, Kelleher K. Preferences for depression advice among low-income women. Matern Child Health J 2003;7:95-102. 10.1023/A:1023864810207 pmid:12870625

31 Lin P, Campbell DG, Chaney EF, et al. The influence of patient preference on depression treatment in primary care. Ann Behav Med 2005:30:16473. 10.1207/s15324796abm3002_9 pmid:16173913.

32 Kwan BM, Dimidjian S, Rizvi SL. Treatment preference, engagement, and clinical improvement in pharmacotherapy versus psychotherapy for depression. Behav Res Ther 2010;48:799-804. 10.1016/j. brat.2010.04.003 pmid:20462569.

33 Battle CL, Howard MM. A mother-baby psychiatric day hospital: History, rationale, and why perinatal mental health is important for obstetric medicine. Obstet Med 2014;7:66-70 10.1177/1753495X13514402 pmid:27512426

34 Dennis CL, Chung-Lee L. Postpartum depression help-seeking barriers and maternal treatment preferences: a qualitative systematic review. Birth 2006;33:323-31. 10.1111/j.1523 536X.2006.00130.x pmid:17150072.

35 Abalovich M, Amino N, Barbour LA, et al. Management of thyroid dysfunction during pregnancy and postpartum: an Endocrine Society Clinical Practice Guideline. / Clin Endocrinol Metab 2007:92(Suppl):S1-47. 10.1210/jc.2007-0141 pmid:17948378.

36 Cassidy-Bushrow AE, Peters RM, Johnson DA, Li J, Rao DS. Vitamin D nutritional status and antenatal depressive symptoms in African American women. J Womens Health (Larchmt) 2012;21:1189-95. 10.1089/ jwh.2012.3528 pmid:22823176.

37 Gur EB, Gokduman A, Turan GA, et al. Mid-pregnancy vitamin D levels and postpartum depression. Eur J Obstet Gynecol Reprod Biol 2014;179:110 6. 10.1016/j.ejogrb.2014.05.017 pmid:24965990

38 Murphy PK, Mueller M, Hulsey TC, Ebeling MD, Wagner CL. An exploratory study of postpartum depression and vitamin d. J Am Psychiatr Nurses Assoc 2010;16:170-7. 10.1177/1078390310370476 pmid:21659271.

39 Corwin E), Murray-Kolb LE, Beard JL. Low hemoglobin level is a risk factor for postpartum depression. J Nutr 2003;133:4139-42. 10.1093/ jn/133.12.4139 pmid:14652362. 
40 Qadri MI, Mushtaq MB, Qazi I, Yousuf S, Rashid A. Sheehan's Syndrome Presenting as Major Depressive Disorder. Iran I Med Sci 2015;40:736.pmid:25648343.

41 Heise AM, Wiessinger D. Dysphoric milk ejection reflex: A case report. Int Breastfeed J 2011;6:6. 10.1186/1746-4358-6-6 pmid:21645333.

42 Wright TE, Terplan M, Ondersma SJ, et al. The role of screening, brief intervention, and referral to treatment in the perinatal period. Am J Obstet Gynecol 2016:215:539-47. 10.1016/J. ajog.2016.06.038 pmid:27373599.

43 Akkaya C, Kocagoz SZ, Turan OF, Taskapilioglu O, Kirli S. Onset of multiple sclerosis following post-partum depressive and manic episodes. Psychiatry Clin Neurosci 2007;61:698-9. 10.1111/j.14401819.2007.01733x pmid.18081637.

44 Sockol LE. A systematic review of the efficacy of cognitive behavioral therapy for treating and preventing perinatal depression. J Affect Disord 2015;177:7-21.10.1016/j.jad.2015.01.052 pmid:25743368

45 Miniati M, Callari A, Calugi S, et al. Interpersonal psychotherapy for postpartum depression: a systematic review. Arch Womens Ment Health 2014:17:257-68.10.1007/s00737-014-0442-7 pmid:24957781.

46 Furuta M, Horsch A, Ng ESW, Bick D, Spain D, Sin J. Effectiveness of trauma-focused psychological therapies for treating post-traumatic stress disorder symptoms in women following childbirth: a systematic review and meta-analysis. Front Psychiatry 2018;9:591. 10.3389/ fpsyt.2018.00591 pmid:30515108.

47 Cohen MJ, Schiller CE. A theoretical framework for treating perinatal depression using couple-based interventions. Psychotherapy (Chic) 2017:54:406-15. 10.1037/pst0000151 pmid:29251961.

48 Newman L, Stevenson C. Issues in infant--parent psychotherapy for mothers with borderline personality disorder. Clin Child Psychol Psychiatry 2008;13:505-14 10.1177/1359104508096766 pmid:18927137.

49 Apter-Danon G, Candilis-Huisman D. A challenge for perinatal psychiatry: therapeutic management of maternal borderline personality disorder an their very young infants. Clin Neuropsychiatry 2005;2:302-14.

50 Tomfohr-Madsen LM, Clayborne ZM, Rouleau CR, Campbell TS. Sleeping for Two: An Open-Pilot Study of Cognitive Behavioral Therapy for Insomnia in Pregnancy. Behav Sleep Med 2017;15:377-93. 10.1080/15402002.2016.1141769 pmid:27124405

51 Rezaei E, Moghadam ZB, Nejat S, Dehghannayeri N. The impact of sleep healthy behavior education on the quality of life in the pregnant women with sleep disorder: A randomized control trial in the year 2012. Iran I Nurs Midwifery Res 2014;19:508-16.pmid:25400680.

52 Dimidjian S, Goodman SH, Sherwood NE, et al. A pragmatic randomized clinical trial of behavioral activation for depressed pregnant women. / Consult Clin Psychol 2017;85:26-36. 10.1037| ccp0000151 pmid:28045285.

53 Lau Y, Htun TP, Wong SN, Tam WSW, Klainin-Yobas P. Therapist-Supported Internet-Based Cognitive Behavior Therapy for Stress, Anxiety, and Depressive Symptoms Among Postpartum Women: A Systematic Review and Meta-Analysis. J Med Internet Res 2017;19:e138. 10.2196/ mir.6712 pmid.28455276.

54 Posmontier B, Neugebauer R, Stuart S, Chittams J, Shaughnessy R. Telephone-Administered Interpersonal Psychotherapy by Nurse-Midwives for Postpartum Depression. J Midwifery Womens Health 2016;61:456 66. 10.1111/jmwh.12411 pmid:26970401

55 Dennis CL, Ravitz P, Grigoriadis S, et al. The effect of telephone-based interpersonal psychotherapy for the treatment of postpartum depression: study protocol for a randomized controlled trial. Trials 2012;13:38. 10.1186/1745-6215-13-38 pmid:22515528.

56 Bloch M, Daly RC, Rubinow DR. Endocrine factors in the etiology of postpartum depression. Compr Psychiatry 2003;44:234-46. 10.1016 S0010-440X(03)00034-8 pmid.12764712.

57 Schiller CE, O'Hara MW, Rubinow DR, Johnson AK. Estradiol modulates anhedonia and behavioral despair in rats and negative affect in a subgroup of women at high risk for postpartum depression. Physiol Behav 2013;119:137-44.10.1016/j.physbeh.2013.06.009 pmid:23770328.

58 Dubey N, Hoffman JF, Schuebel K, et al. The ESC/E(Z) complex, an effector of response to ovarian steroids, manifests an intrinsic difference in cells from women with premenstrual dysphoric disorder. Mol Psychiatry 2017;22:1172-84.10.1038/mp.2016.229 pmid:28044059.

59 Garcia-Esteve L, Navarro P, Ascaso C, et al. Family caregiver role and premenstrual syndrome as associated factors for postnatal depression. Arch Womens Ment Health 2008;11:193-200. 10.1007/s00737-0080012-y pmid:18506575.

60 Bloch M, Rotenberg N, Koren D, Klein E. Risk factors associated with the development of postpartum mood disorders. J Affect Disord 2005;88:918.10.1016/j.jad.2005.04.007 pmid:15979150.

61 Schaffir|, Worly BL, GurTL. Combined hormonal contraception and its effects on mood: a critical review. Eur J Contracept Reprod Health Care 2016;21:347-55 10.1080/13625187.2016.1217327 pmid:27636867

62 Pagano HP, Zapata LB, Berry-Bibee EN, Nanda K, Curtis KM. Safety of hormonal contraception and intrauterine devices among women with depressive and bipolar disorders: a systematic review. Contraception 2016;94:641-9.10.1016/j. contraception.2016.06.012 pmid:27364100.

63 Skovlund CW, Mørch LS, Kessing LV, Lidegaard $\emptyset$. Association of Hormonal Contraception With Depression. JAMA Psychiatry 2016;73:1154-62. 10.1001/jamapsychiatry.2016.2387 pmid:27680324
64 Curtis KM, Tepper NK, Jatlaoui TC, et al. U.S. Medical Eligibility Criteria for Contraceptive Use, 2016. MMWR Recomm Rep 2016;65:1 103.pmid: 27467196

65 Dennis CL, Ross LE, Herxheimer A. Oestrogens and progestins for preventing and treating postpartum depression. Cochrane Database Syst Rev 2008;(4):CD001690.pmid:18843619.

66 HammarbäckS, Bäckström T. Induced anovulation as treatment of premenstrual tension syndrome. A double-blind cross-over study with GnRH-agonist versus placebo. Acta Obstet Gynecol Scand 1988;67:15966. 10.3109/00016348809004191 pmid:3140572.

67 Segebladh B, Borgström A, Nyberg S, Bixo M, Sundström-Poromaa I. Evaluation of different add-back estradiol and progesterone treatments to gonadotropin-releasing hormone agonist treatment in patients with premenstrual dysphoric disorder. Am J Obstet Gynecol 2009·201:139. e1-8.10.1016/jajog 2009.03 .016 pmid:19398092.

68 Guiloff E, Ibarra-Polo A, Zañartu J, Toscanini C, Mischler TW, GómezRogers C. Effect of contraception on lactation. Am J Obstet Gynecol 1974;118:42-5. 10.1016/S0002-9378(16)33643-2 pmid:4128673.

69 Hellgren C, Åkerud H, Skalkidou A, Bäckström T, Sundström-Poromaa I. Low serum allopregnanolone is associated with symptoms of depression in late pregnancy. Neuropsychobiology 2014;69:147-53. 10.1159/000358838 pmid:24776841.

70 Osborne LM, Gispen F, Sanyal A, Yenokyan G, Meilman S, Payne JL. Lower allopregnanolone during pregnancy predicts postpartum depression: An exploratory study. Psychoneuroendocrinology 2017:79:116-21. 10.1016/j.psyneuen.2017.02.012 pmid:28278440.

71 Meltzer-Brody S, Colquhoun H, Riesenberg R, et al. Brexanolone injection in post-partum depression: two multicentre, double-blind, randomised, placebo-controlled, phase 3 trials. Lancet 2018;392:1058-70. 10.1016 S0140-6736(18)31551-4 pmid.30177236.

72 Gelaye B, Addae G, Neway B, et al. Poor sleep quality, antepartum depression and suicidal ideation among pregnant women. J Affect Disord 2017;209:195-200. 10.1016/j. jad.2016.11.020 pmid:27930912.

73 Okun ML. Disturbed Sleep and Postpartum Depression. Curr Psychiatry Rep 2016;18:66. 10.1007/s11920-016-0705-2 pmid:27222140.

74 BaHammam AS, Kendzerska T, Gupta R, et al. Comorbid depression in obstructive sleep apnea: an under-recognized association. Sleep Breath 2016;20:447-56. 10.1007/s11325-015-1223-x pmid:26156890.

75 Edwards C, Mukheriee S, Simpson L, Palmer LI, Almeida OP, Hillman DR. Depressive Symptoms before and after Treatment of Obstructive Sleep Apnea in Men and Women. J Clin Sleep Med 2015;11:102938.pmid:25902824.

76 Borak J, Cieślicki JK, Koziej M, Matuszewski A, Zieliński J. Effects of CPAP treatment on psychological status in patients with severe obstructive sleep apnoea. / Sleep Res 1996:5:123-7. 10.1046/j.1365-2869.1996. d01-60.x pmid:8795813.

77 Sharkey KM, Boni GM, Quattrucci JA, Blatch S, Carr SN. Women with Postpartum Weight Retention Have Delayed Wake Times and Decreased Sleep Efficiency During the Perinatal Period: A Brief Report. Sleep Health 2016;2:225-8. 10.1016/j.sleh.2016.05.002 pmid:27857964.

78 Taylor DJ, Pruiksma KE. Cognitive and behavioural therapy for insomnia (CBT-I) in psychiatric populations: a systematic review. Int Rev Psychiatry 2014;26:205-13 10.3109/09540261.2014.902808 pmid:24892895.

79 Swanson LM, Flynn H, Adams-Mundy JD, Armitage R, Arnedt JT. An open pilot of cognitive-behavioral therapy for insomnia in women with postpartum depression. Behav Sleep Med 2013;11:297-307. 10.1080/15402002.2012.683902 pmid:23216373

80 Research on Expecting moms and Sleep Therapy (REST) Study. Research on Expecting Moms and Sleep Therapy (REST). 2016. https://rest.ucsf. edu/.

81 Douglas PS, Hill PS. Behavioral sleep interventions in the first six months of life do not improve outcomes for mothers or infants: a systematic review. J Dev Behav Pediatr 2013;34:497-507. 10.1097/ DBP.0b013e31829cafa6 pmid:24042081.

82 Hiscock H, Cook F, Bayer I, et al. Preventing early infant sleep and crying problems and postnatal depression: a randomized trial. Pediatrics 2014;133:e346-54. 10.1542/peds.2013-1886 pmid:24394682

83 Phillips J, Sharpe L, Nemeth D. Maternal psychopathology and outcomes of a residential mother-infant intervention for unsettled infant behaviour. Aust N Z J Psychiatry 2010;44:280-9. 10.3109/00048670903487225 pmid:20180728.

84 Smart J, Hiscock H. Early infant crying and sleeping problems: a pilot study of impact on parental well-being and parent-endorsed strategies for management. J Paediatr Child Health 2007;43:284-90. 10.1111/j.14401754.2007.01060.x pmid:17444831.

85 Stremler R, Hodnett E, Lee K, et al. A behavioral-educational intervention to promote maternal and infant sleep: a pilot randomized, controlled trial. Sleep 2006;29:1609-15.10.1093/ sleep/29.12.1609 pmid:17252892.

86 Stremler R, Hodnett E, Kenton L, et al. Effect of behavioural-educational intervention on sleep for primiparous women and their infants in early postpartum: multisite randomised controlled trial. BMJ 2013:346:f1164. 10.1136/bmj.f1164 pmid:23516146.

87 Wirz-Justice A, Bader A, Frisch U, et al. A randomized, doubleblind, placebo-controlled study of light therapy for antepartum depression. J Clin Psychiatry 2011;72:986-93. 10.4088/ JCP.10m06188blu pmid:21535997. 
88 Corral M, Wardrop AA, Zhang H, Grewal AK, Patton S. Morning light therapy for postpartum depression. Arch Womens Ment Health 2007;10:221-4. 10.1007/s00737-007-0200-1 pmid:17701271.

89 Swanson LM, Burgess HJ, Zollars J, Todd Arnedt J. An open-label pilo study of a home wearable light therapy device for postpartum depression. Arch Womens Ment Health 2018;21:583-6. 10.1007/s00737-0180836-z pmid:29603017.

90 Bifulco A Figueiredo B, Guedeney N, et al TCS-PND Group Maternal attachment style and depression associated with childbirth: preliminary results from a European and US cross-cultural study. BrJ Psychiatry Supp 2004:46:s31-7.10.1192/bjp.184.46.s31 pmid:14754816

91 Ikeda M, Hayashi M, Kamibeppu K. The relationship between attachmen style and postpartum depression. Attach Hum Dev 2014;16:557-72. 10.1080/14616734.2014.941884 pmid:25098625.

92 Robakis TK, Williams KE, Crowe S, Lin KW, Gannon J, Rasgon NL. Maternal attachment insecurity is a potent predictor of depressive symptoms in the early postnatal period. J Affect Disord 2016;190:623-31.10.1016/j. jad.2015.09.067 pmid:26583352.

93 Grant KA, Bautovich A, McMahon C, Reilly N, Leader L, Austin MP. Parenta care and control during childhood: associations with maternal perinatal mood disturbance and parenting stress. Arch Womens Ment Health 2012;15:297-305.10.1007/s00737-012-0292-0 pmid:22695807.

94 Tani F, Castagna V, Ponti L. Women who had positive relationships with their own mothers reported good attachments to their first child before and after birth. Acta Paediatr 2018;107:633-7. 10.1111/ apa.14162 pmid:29172238.

95 Strupp H, et al. Psychotherapy in a new key.Basic Books, 1984

96 Travis LA, Bliwise NG, Binder JL, Horne-Moyer HL. Changes in clients' attachment styles over the course of time-limited dynamic psychotherapy. Psychotherapy 2001:38:149-5910.1037/0033-3204.38.2.149.

97 Miniati M, Callari A, Calugi S, et al. Interpersonal psychotherapy for postpartum depression: a systematic review. Arch Womens Ment Health 2014;17:257-68. 10.1007/s00737-014-0442-7 pmid:24957781.

98 Giallo R, Cooklin A, Nicholson JM. Risk factors associated with trajectories of mothers' depressive symptoms across the early parenting period: an Australian population-based longitudinal study. Arch Womens Ment Health 2014;17:115-25. 10.1007/s00737-014-0411 1 pmid:24424796.

99 Hudson C, Spry E, Borschmann R, et al. Preconception personality disorder and antenatal maternal mental health: A populationbased cohort study. J Affect Disord 2017;209:169-76. 10.1016/j. jad.2016.11.022 pmid:27923193.

100 Uguz F, Akman C, Sahingoz M, Kaya N, Kucur R. One year follow-up of post-partum-onset depression: the role of depressive symptom severity and personality disorders. I Psychosom Obstet Gynaecol 2009;30:141-5. 10.1080/01674820802545818 pmid:19533495.

101 Macfie J. Development in Children and Adolescents Whose Mothers Have Borderline Personality Disorder. Child Dev Perspect 2009;3:66. 10.1111/j.1750-8606.2008.00079.x pmid:20161670.

102 Huntley F, Wright N, Pickles A, Sharp H, Hill J. Maternal mental health and child problem behaviours: disentangling the role of depression and borderline personality dysfunction. BJPsych Open 2017:3:300-5. 10.1192/bjpo.bp.117.005843 pmid:29234522.

103 Pare-Miron V, Czuzoj-Shulman N, Oddy L, Spence AR, Abenhaim HA Effect of borderline personality disorder on obstetrical and neonatal outcomes. Womens Health Issues 2016;26:190-5.10.1016/j. whi.2015.11.001 pmid:26718528.

104 Gorwood P, Rouillon F, Even C, Falissard B, Corruble E, Moran P. Treatment response in major depression: effects of personality dysfunction and prior depression. BrJ Psychiatry 2010;196:139-42.10.1192/bjp. bp.109.067058 pmid:20118460.

105 Goddard E, Wingrove J, Moran P. The impact of comorbid personality difficulties on response to IAPT treatment for depression and anxiety. Behav Res Ther 2015;73:1-7. 10.1016/j. brat.2015.07.006 pmid:26226089.

106 Cohen N, et al. Watch, Wait, and Wonder: Testing the Effectiveness of a New Approach to Mother-Infant Psychotherapy. Infant Ment Health I 1999:20:429-5110.1002/(SICI)1097-0355(199924)20:4<429*:AIDIMHI5>3.0.CO;2-0.

107 Newman L, Stevenson C. Issues in infant--parent psychotherapy for mothers with borderline personality disorder. Clin Child Psychol Psychiatry 2008:13:505-14. 10.1177/1359104508096766 pmid:18927137.

108 Lydsdottir LB, Howard LM, Olafsdottir H, Thome M, Tyrfingsson P, Sigurdsson JF. The mental health characteristics of pregnant women with depressive symptoms identified by the Edinburgh Postnatal Depression Scale. / Clin Psychiatry 2014;75:393-8. 10.4088/ ICP.13m08646 pmid:24569071.

109 Leigh B, Milgrom J. Risk factors for antenatal depression, postnatal depression and parenting stress. BMC Psychiatry 2008;8:24. 10.1186/1471-244X-8-24 pmid:18412979.

110 Melville JL, Gavin A, Guo Y, Fan MY, Katon WJ. Depressive disorders during pregnancy: prevalence and risk factors in a large urban sample. Obstet Gynecol 2010;116:1064-70. 10.1097/ AOG 0b013e3181f60b0a pmid:20966690.

111 Martini J, Petzoldt J, Einsle F, Beesdo-Baum K, Höfler M, Wittchen HU. Risk factors and course patterns of anxiety and depressive disorders during pregnancy and after delivery: a prospectivelongitudinal study. J Affect Disord 2015;175:385-95.10.1016/j. jad.2015.01.012 pmid:25678171.
112 Agostini F, Neri E, Salvatori P, Dellabartola S, Bozicevic L, Monti F. Antenatal depressive symptoms associated with specific life events and sources of social support among Italian women. Matern Child Health I 2015;19:1131-41. 10.1007/s10995-014-1613-x pmid:25303805.

113 Räisänen S, Lehto SM, Nielsen HS, Gissler M, Kramer MR, Heinonen S. Risk factors for and perinatal outcomes of major depression during pregnancy: a population-based analysis during 2002 2010 in Finland. BMJ Open 2014;4:e004883. 10.1136/ bmjopen-2014-004883 pmid:25398675.

114 Sockol LE, Epperson CN, Barber JP. Preventing postpartum depression: a meta-analytic review. Clin Psychol Rev 2013;33:1205-17. 10.1016/j. cpr.2013.10.004 pmid:24211712.

115 Cuiipers P, Brännmark JG, van Straten A. Psychological treatment of postpartum depression: a meta-analysis. J Clin Psychol 2008;64:103-18. 10.1002/jclp.20432 pmid:18161036.

116 O'Mahen HA, Woodford J, McGinley J, et al. Internet-based behaviora activation--treatment for postnatal depression (Netmums): a randomized controlled trial. J Affect Disord 2013;150:814-22. 10.1016/j. jad.2013.03.005 pmid:23602514

117 Dimidjian S, Goodman SH, Felder JN, Gallop R, Brown AP, Beck A. An open trial of mindfulness-based cognitive therapy for the prevention of perinatal depressive relapse/recurrence. Arch Womens Ment Health 2015;18:85-94. 10.1007/s00737-014-0468-x pmid:25298253.

118 Tezel A, Gözüm S. Comparison of effects of nursing care to problem solving training on levels of depressive symptoms in post partum women. Patient Educ Couns 2006;63:64-73.10.1016/j. pec.2005.08.011 pmid:16448799.

119 Scope A, Leaviss J, Kaltenthaler E, et al. Is group cognitive behaviour therapy for postnatal depression evidence-based practice? A systematic review. BMC Psychiatry 2013;13:321. 10.1186/1471-244X-13321 pmid:24283266.

120 Brugha TS, WheatleyS, Taub NA, et al. Pragmatic randomized trial of antenatal intervention to prevent post-natal depression by reducing psychosocial risk factors. Psychol Med 2000;30:1273-81. 10.1017/ S0033291799002937 pmid:11097068.

121 Puckering C, et al. Mellow Babies: A group intervention for infants and mothers experiencing postnatal depression. Couns Psychol Rev 2010;25:28-40

122 Yildiz PD, Ayers S, Phillips L. The prevalence of posttraumatic stress disorder in pregnancy and after birth: A systematic review and meta-analysis. / AffectDisord 2017:208:634-45.10.1016/j. jad.2016.10.009 pmid:27865585.

123 Dindo L, Elmore A, O'Hara M, Stuart S. The comorbidity of Axis I disorders in depressed pregnant women. Arch Womens Ment Health 2017;20:757 64. 10.1007/s00737-017-0769-y pmid:28842756.

124 Dikmen-Yildiz P. Ayers S, Phillips L. Depression, anxiety, PTSD and comorbidity in perinatal women in Turkey: A longitudinal population-based study. Midwifery 2017;55:29-37. 10.1016/j. midw.2017.09.001 pmid:28917088.

125 Hurlocker MC, Vidaurri DN, Cuccurullo LJ, Maieritsch K, Franklin CL. Examining the latent structure mechanisms for comorbid posttraumatic stress disorder and major depressive disorder. J Affect Disord 2018;229:477-82. 10.1016/j.jad.2017.12.076 pmid:29334642.

126 Grote NK, Katon WJ, Russo JE, et al. A Randomized Trial of Collaborative Care for Perinatal Depression in Socioeconomically Disadvantaged Women: The Impact of Comorbid Posttraumatic Stress Disorder / Clin Psychiatry 2016:77:1527-37. 10.4088/ JCP.15m10477 pmid:28076671.

127 Lenze SN, Potts MA. Brief Interpersonal Psychotherapy for depression during pregnancy in a low-income population: A randomized controlled trial. / Affect Disord 2017;210:151-7.10.1016/j. jad.2016.12.029 pmid:28038377.

128 Interian A, Lewis-Fernández R, Dixon LB. Improving treatment engagement of underserved U.S. racial-ethnic groups: a review of recent interventions. Psychiatr Serv 2013;64:212-22. 10.1176/appi. ps.201100136 pmid:23203442

129 Watts BV, Schnurr PP, Mayo L, Young-Xu Y, Weeks WB, Friedman M]. Meta-analysis of the efficacy of treatments for posttraumatic stress disorder. J Clin Psychiatry 2013;74:e541-50. 10.4088/ JCP.12r08225 pmid:23842024

130 Lee DJ, Schnitzlein CW, Wolf JP, Vythilingam M, Rasmusson AM, Hoge CW. Psychotherapy Versus Pharmacotherapy for Posttraumatic Stress Disorder: Systemic Review and Meta-Analyses to Determine FirstLine Treatments. Depress Anxiety 2016;33:792-806. 10.1002/ da.22511 pmid:27126398

131 Resick PA, Nishith P, Weaver TL, Astin MC, Feuer CA. A comparison of cognitive-processing therapy with prolonged exposure and a waiting condition for the treatment of chronic posttraumatic stress disorder in female rape victims. J Consult Clin Psychol 2002;70:867-79. 10.1037/0022-006X.70.4.867 pmid:12182270.

132 Stein DJ, Seedat S, van der Linden GJ, Zungu-Dirwayi N. Selective serotoni reuptake inhibitors in the treatment of post-traumatic stress disorder: a meta-analysis of randomized controlled trials. Int Clin Psychopharmacol 2000;15(Suppl 2):S31-9. 10.1097/00004850-20000800200006 pmid:11110017.

133 Marwood L, Wise T, Perkins AM, Cleare AJ. Meta-analyses of the neural mechanisms and predictors of response to psychotherapy in depression and anxiety. Neurosci Biobehav Rev 2018;95:61-72. 10.1016/j. neubiorev.2018.09.022 pmid:30278195. 
134 Sockol LE. A systematic review and meta-analysis of interpersonal psychotherapy for perinatal women. J Affect Disord 2018;232:316-28. 10.1016/j.jad.2018.01.018 pmid.29501991.

135 Sockol LE. A systematic review of the efficacy of cognitive behavioral therapy for treating and preventing perinatal depression. J Affect Disord 2015;177:7-21.10.1016/j.jad.2015.01.052 pmid:25743368.

136 Cuijpers P, van Straten A, van Oppen P, Andersson G. Are psychological and pharmacologic interventions equally effective in the treatment of adult depressive disorders? A meta-analysis of comparative studies. J Clin Psychiatry 2008;69:1675-85, quiz 1839-41. 10.4088/JCP. v69n1102 pmid:18945396.

137 Dennis CL, Hodnett E. Psychosocial and psychological interventions for treating postpartum depression. Cochrane Database Syst Rev 2007:(4):CD006116.pmid:17943888.

138 Ravitz P, McBride C, Maunder R. Failures in interpersonal psychotherapy (IPT): factors related to treatment resistances. J Clin Psychol 2011;67:112939. 10.1002/jclp.20850 pmid:21968699.

139 Mandelli L, Souery D, Bartova L, et al. Bipolar II disorder as a risk factor for postpartum depression. / Affect Disord 2016;204:54-8. 10.1016/j. jad.2016.06.025 pmid:27327115

140 FrankE, Kupfer DJ, Thase ME, et al. Two-year outcomes fo interpersonal and social rhythm therapy in individuals with bipolar I disorder. Arch Gen Psychiatry 2005;62:996-1004. 10.1001/ archpsyc.62.9.996 pmid:16143731.

141 Pfeiffer PN, Heisler M, Piette JD, Rogers MA, Valenstein M. Efficacy of peer support interventions for depression: a meta-analysis. Gen Hosp Psychiatry 2011;33:29-36. 10.1016/j.genhosppsych.2010.10.002 pmid:21353125.

142 WenzeSJ, Battle CL. Perinatal Mental Health Treatment Needs, Preferences, and Barriers in Parents of Multiples. J Psychiatr Pract 2018;24:158-68. 10.1097/PRA.0000000000000299 pmid:30015786.

143 Ashford MT, Olander EK, Ayers S. Computer- or web-based interventions for perinatal mental health: A systematic review. J Affect Disord 2016;197:13446.10.1016/j.jad.2016.02.057 pmid:26991368

144 Kumar R, Marks M, Platz C, Yoshida K. Clinical survey of a psychiatric mother and baby unit: characteristics of 100 consecutive admissions. / Affect Disord 1995;33:11-22.10.1016/0165-0327(94)00067-J pmid:7714304.

145 Oates M. Psychiatric services for women following child-birth. Int Rev Psychiatry 1996;8:87-9810.3109/09540269609037821.

146 Howard M, Battle CL, Pearlstein T, Rosene-Montella K. A psychiatric motherbaby day hospital for pregnant and postpartum women. Arch Womens Ment Health 2006;9:213-8. 10.1007/s00737-006-0135-y pmid:16718517.
147 Meltzer-Brody S, Brandon AR, Pearson B, et al. Evaluating the clinical effectiveness of a specialized perinatal psychiatry inpatient unit Arch Womens Ment Health 2014:17:107-13, 10.1007/s00737-013-03907 pmid:24201978.

148 Kim DR, Epperson N, Paré E, et al. An open label pilot study of transcranial magnetic stimulation for pregnant women with major depressive disorder. / Womens Health (Larchmt) 2011;20:255-61. 10.1089/iwh.2010.2353 pmid:21314450.

149 Hızlı Sayar G, Ozten E, Tufan E, et al. Transcranial magnetic stimulation during pregnancy. Arch Womens Ment Health 2014;17:311-5. 10.1007/ s00737-013-0397-0 pmid:24248413.

150 Zhang D, Hu Z. RTMS may be a good choice for pregnant women with depression. Arch Womens Ment Health 2009;12:189-90. 10.1007/ s00737-009-0058-5 pmid:19238519.

151 Zhang X, Liu K, Sun J, Zheng Z. Safety and feasibility of repetitive transcranial magnetic stimulation (rTMS) as a treatment for major depression during pregnancy. Arch Womens Ment Health 2010;13:36970. 10.1007/s00737-010-0163-5 pmid:20386939.

152 Klirova M, Novak T, Kopecek M, Mohr P, Strunzova V. Repetitive transcranial magnetic stimulation (rTMS) in major depressive episode during pregnancy. Neuro Endocrinol Lett 2008;29:69 70.pmid: 18283246

153 Garcia KS, Flynn P, Pierce KJ, Caudle M. Repetitive transcranial magnetic stimulation treats postpartum depression. Brain Stimul 2010;3:36-41. 10.1016/j.brs.2009.06.001 pmid:20633429.

154 Yanamadala J, et al. Estimates of peak electric fields induced by Transcranial magnetic stimulation in pregnant women as patients using an FEM full-body model. 39th Annual International Conference of the IEEE Engineering in Medicine and Biology Society 2017:1441-4.

155 Gahr M, Blacha C, Connemann BJ, Freudenmann RW, SchönfeldtLecuona C. Successful treatment of major depression with electroconvulsive therapy in a pregnant patient with previous nonresponse to prefrontal rTMS. Pharmacopsychiatry 2012;45:79-80 10.1055/s-0031-1297936 pmid:22174028.

156 Rasmussen KG. Some considerations in choosing electroconvulsive therapy versus transcranial magnetic stimulation for depression. J ECT 2011;27:51-4. 10.1097/YCT.0b013e3181da84c6 pmid:21343711.

157 Molenaar NM, Kamperman AM, Boyce P, Bergink V. Guidelines on treatment of perinatal depression with antidepressants: An international review. Aust N Z J Psychiatry 2018;52:320-7. 10.1177/0004867418762057 pmid:29506399. 